Pacific

Journal of

Mathematics

ON THE EXISTENCE OF SPECIAL STABLE SPANNED VECTOR BUNDLES ON PROJECTIVE CURVES

\author{
E. BALLICO
}




\title{
ON THE EXISTENCE OF SPECIAL STABLE SPANNED VECTOR BUNDLES ON PROJECTIVE CURVES
}

\author{
E. BALLico
}

Let $X$ be a smooth projective curve. Here we give conditions on $r, d, v$ (essentially, existence of special spanned line bundles on $X$ with many sections) for the existence of a rank $r$ spanned vector bundle $E$ on $X$ with $\operatorname{rank}(E)=r, \operatorname{deg}(E)$ $=d$ and $h^{0}(X, E) \geq v$.

\section{Introduction.}

Let $X$ be a smooth projective curve of genus $g \geq 2$ defined over an arbitrary algebraically closed field $\mathbf{K}$. For any vector bundle $E$ on $X$, call $\mu(E):=$ $\operatorname{deg}(E) / \operatorname{rank}(E)$ the slope of $E$. A vector bundle $E$ on $X$ is said to be spanned if the natural map $H^{0}(X, E) \otimes O_{X} \rightarrow E$ is surjective. Such bundles are important tools for the projective geometry of $X$ because they are exactly the vector bundles associated to a morphism from $X$ into a Grassmannian. A vector bundle $E$ on $X$ is said to be stable (resp. semistable) if for every proper subsheaf $A$ of $E$ we have $\mu(A)<\mu(E)$ (resp. $\mu(A) \leq \mu(E)$ ). In particular every line bundle is stable. A stable line bundle, $E$, on $X$ is simple, i.e., every endomorphism of $E$ is induced by the multiplication by some $\lambda \in \mathbf{K}$. The stable vector bundles on $X$ are the more interesting bundles on $X$ from the point of view of moduli problems. The aim of this paper is the proof of the following result.

Theorem 0.1. Let $X$ be a smooth projective curve of genus $g \geq 2$. Fix integers $r, k, m$ with $r \geq 2, k \geq 2$ and $m>0$. Assume the existence of line bundles $L_{i} \in \operatorname{Pic}^{k}(X), 1 \leq i \leq r, M_{u} \in \operatorname{Pic}^{k+1}(X), 1 \leq u<r$, with the following properties:

(a) Every $L_{i}, 1 \leq i \leq r$, and every $M_{u}, 1 \leq u<r$, is spanned;

(b) $h^{0}\left(X, L_{i}\right) \geq m$ for every integer $i$ with $1 \leq i \leq r$ and $h^{0}\left(X, M_{u}\right) \geq$ $m+1$ for every integer $u$ with $1 \leq u<r$;

(c) no two of the line bundles $L_{i}, 1 \leq i \leq r$, are isomorphic;

(d) no two of the line bundles $M_{u}, 1 \leq u<r$, are isomorphic;

(e) we have $h^{0}\left(X, \operatorname{Hom}\left(L_{i}, M_{u}\right)\right)=0$ for every pair $(i, u)$ with $1 \leq i \leq r$ and $1 \leq u<r$, i.e., for every pair $(i, u)$ there is no $P \in X$ with $M_{u} \cong L_{i}(P)$. 
Then for every integer $d$ with $r k<d<r(k+1)$ there exists a rank $r$ spanned stable vector bundle $E$ on $X$ with $\operatorname{deg}(E)=d$ and $h^{0}(X, E) \geq m r$.

Remark 0.2. Fix the integers $g, r, m$ with $g \geq 2, r \geq 2$ and $m>0$. Let $\rho(g, y, x):=g-(x+1)(g+x-y)$ be the Brill-Noether number for $g_{y}^{x}$ 's on curves of genus $g$. Let $X$ be a general curve of genus $g$. By Brill-Noether theory $([\mathbf{1}], \mathrm{Ch}$. V and Ch. VII), the assumptions of 0.1 are satisfied for the triple $(r, k, m)$ if and only if we have $\rho(g, m, k) \geq 0$ and either $\rho(g, m, k)>0$ or the finite number (computed in [1], p. 211), of $g_{k}^{m}$ 's on $X$ is at least $r$.

Remark 0.3. We believe that Theorem 0.1 is quite strong. Its main feature is that its statement depends only on the Brill-Noether theory of special line bundles on $X$. The problem of existence of spanned stable vector bundles on $X$ is quite different from the problem of existence of stable vector bundles on $X$ with many sections. For instance if $2 d \geq g+2$ there exists $L \in \operatorname{Pic}^{d}(X)$ with $h^{0}(X, L) \geq 2([\mathbf{1}])$; however, if $X$ is hyperelliptic and $d \leq g+1 \operatorname{such} L$ may be spanned only if $d$ is even. There are curves of genus $g$ for which the assumptions of 0.1 are not satisfied for a certain triple $(r, k, m)$, while by Remark 0.2 for the same integers the assumptions of 0.1 are satisfied for a general curve of genus $g$ and the same triple $(r, k, m)$. For instance on a hyperelliptic curve there is no spanned special line bundle of odd degree. This phenomenon is not due to the weakness of 0.1 but to the nature of the problem of existence of spanned vector bundles. This phenomenon arises even for line bundles, as shown by the case of hyperelliptic curves. Conversely, there are curves not with general moduli for which 0.1 gives a better result than the on the corresponding one on a general curve. For any smooth curve $C$ the set of nonnegative integers $k$ such that $C$ has a base point free $g_{k}^{1}$ is called the Lüroth semigroup of $C$. See [4] for the computation of the Lüroth semigroup of smooth plane curves and [6] for the computation of the Lüroth semigroup of a general $k$-gonal curve of genus $g$. See the introductions and the references of [4] and [6] for several other results on this topic. As stressed in Remarks 0.2 and 0.3 the existence of special spanned line bundles on $X$ depends on $X$, not just on its genus, even in the range of integers $d, x$ with $\rho(g, d, x) \geq 0$ in which by the classical existence theorem of Brill-Noether theory ([1] , Ch. V and Ch. VII) there is $L \in \operatorname{Pic}^{d}(X)$ with $h^{0}(X, L) \geq x+1$. For line bundles the spannedness condition is important because only these line bundles correspond to morphisms from $X$ to projective spaces. Similarly, only spanned vector bundles corresponds to morphisms from $X$ into Grassmannians. If $L \in \operatorname{Pic}(X)$ has many sections, at least throwing away the base locus, $B$, we obtain a spanned line bundle $L(-B)$ with $h^{0}(X, L(-B))=h^{0}(X, L(-B))$ and hence at least a morphism of degree $\operatorname{deg}(L)-\operatorname{length}(B)<\operatorname{deg}(L)$ to the same projective space. But if $E$ is a vector bundle with $r:=\operatorname{rank}(E)>1$ and $E^{\prime}$ is a subsheaf of $E$ spanned by $H^{0}(X, E)$, we may have $\operatorname{rank}\left(E^{\prime}\right)<\operatorname{rank}(E)$. Even if we assume 
$\operatorname{rank}\left(E^{\prime}\right)=\operatorname{rank}(E)$, the geometric properties of $E^{\prime}$ may be very different from the ones of $E$. In particular $E^{\prime}$ may be unstable even if $E$ is stable. For instance as far as we know the sharper existence theorems for stable vector bundles with many sections on $\mathrm{X}$ produce stable bundles, $E$, such that $E^{\prime}$ is a direct sum of line bundles (see e.g [6]) and hence with $E^{\prime}$ not stable, not simple and usually not even semistable. We hope to have convinced the reader that for rank $r>1$ the Brill-Noether theory of stable vector bundles on $X$ and the Brill-Noether theory of stable and spanned vector bundles on $X$ are quite different and that both (but even more the second one) depends on the algebraic structure of $X$, not just its genus. Theorem 0.1 is easy to use both for curves with general moduli and for curves for which the classical theory of special divisor is known. The interested reader may see the case of plane curves in [5]. The proof of 0.1 uses a construction (see 1.1) made in [2] for the same purpose. Except for the use of this construction, the proofs are completely different from the ones in $[2]$.

\section{Proof of 0.1 .}

The following construction of spanned vector bundles was made in [2], Construction 1.1.

Construction 1.1. We fix a vector bundle $A$ on $X$ and a rank $t$ vector bundle $M$ on $X$ which is spanned by its global sections. We fix an integer $x$ with $t+1 \leq x \leq h^{0}(X, M)$. We are looking for exact sequences on $X$ :

$$
0 \rightarrow A \rightarrow E \rightarrow M \rightarrow 0
$$

with $h^{0}(X, E)=h^{0}(X, A)+x$ and such that the image of $H^{0}(X, E)$ into $H^{0}(X, M)$ is an $x$-dimensional subspace of $H^{0}(X, M)$ spanning $M$. Notice that if $A$ is spanned, then any such $E$ is spanned. Here we will construct all such bundles $E$, although in this paper we only use that the construction gives spanned bundles. Fix a linear subspace $W \subseteq H^{0}(X, M)$ with $\operatorname{dim}(W)=x$ and $W$ spanning $M$. We want to find all exact sequences (1) such that the image of the map $H^{0}(X, E) \rightarrow H^{0}(X, M)$ contains $W$. In particular we will obtain $h^{0}(X, E) \geq h^{0}(X, A)+x$. Consider the Euler's sequence on the Grassmannnian $G(t, x)$ seen as the Grassmannian of all $(x-t)$-dimensional linear subspaces of $W$ :

$$
0 \rightarrow S \rightarrow W \otimes O_{G(t, x)} \rightarrow Q \rightarrow 0 .
$$

Since $W$ spans $M$, the universal property of the Grassmannian $G(t, x)$ gives a morphism $f: X \rightarrow G(t, x)$ such that $M \cong f^{*}(Q)$. Set $U:=f^{*}(S)$. Hence $U$ is a vector bundle with $\operatorname{rank}(U)=x-t, \operatorname{deg}(U)=-\operatorname{deg}(M)$ and $U^{*}$ is spanned by $W^{*}$. Thus if $M$ has no trivial factor, we have $h^{0}\left(X, U^{*}\right) \geq x$. The case $x=t+1$ is very nice because in this case we have $U^{*} \cong \operatorname{det}(M)$. Fix $j \in H^{0}(X ; \operatorname{Hom}(U, A))$. The map $j$ induces a map $u(j): U \rightarrow A \oplus O_{X \oplus x}$. 
Recall that a subsheaf $T^{\prime}$ of a locally free sheaf $T$ on $X$ is said to be saturated (or saturated in $T$ ) if $T / T^{\prime}$ has no torsion, i.e., if either $T^{\prime}=T$ or $T / T^{\prime}$ is locally free. Since $W$ spans $M, U$ is a saturated subsheaf of $W \otimes O_{X}$ and $u(j)(U)$ is saturated in $A \oplus O_{X^{\oplus x}}$. Hence Coker $(u(j))$ is a vector bundle. Set $E:=\operatorname{Coker}(u(j))$. We have $\operatorname{rank}(E)=\operatorname{rank}(A)+\operatorname{rank}(M)$. By construction $E$ has $A$ as a saturated subbundle. By construction $E$ fits in an exact sequence (1). By construction the $x$ chosen spanning sections of $M$ are lifted to $E$. Now we check that this construction gives all such bundles. Take $E$ fitting in (1) with $h^{0}(X, E)=h^{0}(X, A)+x$. Hence the image, $W$, of $H^{0}(X, E)$ into $H^{0}(X, M)$ has rank $x$; if $E$ is spanned, then $W$ spans $M$ and $E$ is a quotient of $A \oplus W$ with $U$ as kernel, i.e., we have an exact sequence

$$
0 \rightarrow U \rightarrow A \oplus W \rightarrow E \rightarrow 0
$$

in which the map $U \rightarrow W$ is induced by (2), while the map $U \rightarrow A$ obtained from (3) is our map $j$.

Lemma 1.2. Fix an integer $r \geq 2$. Let $X$ be a smooth curve of genus $\geq 1$. Fix $L_{i} \in \operatorname{Pic}(X), 1 \leq i \leq r-1$, and $M \in \operatorname{Pic}(X)$ with $\operatorname{deg}\left(L_{i}\right)=\operatorname{deg}(M)-1$ for every $i$. Assume $L_{i} \cong L_{k}$ if and only if $i=k$ and $h^{0}\left(X, \operatorname{Hom}\left(L_{i}, M\right)\right)=0$ for every integer $i$ with $1 \leq i \leq r-1$ i.e., assume that there is no $P \in X$ with $M \cong L_{i}(P)$ for some $i$. Let $E$ be a rank $r$ vector bundle on $X$ fitting in an exact sequence (1) with $A=L_{1} \oplus \cdots \oplus L_{r-1}$. Then $E$ is stable if and only if it has no $L_{i}$ as a direct factor.

Proof. Assume the existence of a proper subbundle $F$ of $E$ with $\mu(E) \leq$ $\mu(F)$. Taking $F$ with minimal rank we may assume $F$ stable. Since $\mu(E)=$ $\operatorname{deg}\left(L_{1}\right)+1 / r$ and $\operatorname{rank}(F)<r$, we obtain $\mu(F) \geq \operatorname{deg}\left(L_{1}\right)=\operatorname{deg}(M)-1$. Hence the stability of $F$ implies that either the induced map $f: F \rightarrow M$ is zero or it is surjective. Since $A$ is semistable and $\mu(F)>\mu(A), F$ is not contained in $A$. Hence $f$ is surjective. Since $\operatorname{Ker}(f) \subseteq A$, either $\operatorname{Ker}(f)$ is a direct sum of some of the $L_{j}$ 's or $\operatorname{deg}(F)=\operatorname{deg}(M)+\operatorname{deg}(\operatorname{Ker}(f)) \leq$ $\operatorname{rank}(f)\left(\operatorname{deg}\left(L_{1}\right)\right)$. Thus $\operatorname{Ker}(f)$ is a direct sum of some of the $L_{j}$ 's, say $\operatorname{Ker}(f) \cong \oplus_{1 \leq i \leq s} L_{i}$ with $1 \leq s \leq r-2$. Thus $\mu(E / F)=\operatorname{deg}\left(L_{1}\right)$. Since $A$ is semistable, we obtain the existence of an integer $i$ with $1 \leq i \leq r-1$ such that the compositions of the maps $L_{i} \rightarrow A \rightarrow E \rightarrow E / F \rightarrow L_{s+1}$ induce a splitting of $E$ with $L_{i}$ as direct factor.

Corollary 1.3. Let $X$ be a smooth curve of genus $\geq 1$. Fix $L_{i} \in \operatorname{Pic}(X)$, $1 \leq i \leq r-1$, and $M \in \operatorname{Pic}(X)$ with $\operatorname{deg}\left(L_{i}\right)=\operatorname{deg}(M)-1$ for every $i$. Assume $L_{i} \cong L_{k}$ if and only if $i=k$ and $h^{0}\left(X, \operatorname{Hom}\left(L_{i}, M\right)\right)=0$ for every integer $i$ with $1 \leq i \leq r-1$ i.e., assume that there is no $P \in X$ with $M \cong L_{i}(P)$ for some $i$. Set $A:=L_{1} \oplus \cdots \oplus L_{r-1}$. Assume $h^{0}\left(X, L_{i}\right) \geq 2$ for every $i$ and $M$ spanned. Assume $L_{i} \cong L_{k}$ if and only if $i=k$ and $h^{0}\left(X, \operatorname{Hom}\left(L_{i}, M\right)\right)=0$ for every integer $i$ with $1 \leq i \leq r-1$ i.e., assume that there is no $P \in X$ with $M \cong L_{i}(P)$ for some $i$. Fix a linear space 
$W \subseteq H^{0}(X, M)$ with $\operatorname{dim}(W)=2$ and $W$ spanning $M$. Let $E$ be the general spanned bundle obtained by Construction 1.1 with respect to the vector space $W$. Then $E$ is stable.

Proof. Since $\operatorname{dim}(W)=2$, we have $U \cong M^{*}$. Since $h^{0}\left(X, L_{i}\right) \geq 2$, we have $h^{0}\left(X, \operatorname{Hom}\left(U, L_{i}\right)\right) \geq 3$. Hence for a general $j \in H^{0}(X, \operatorname{Hom}(U, A))$ the bundle Coker $(u(j))$ has no $L_{i}$ as a direct factor. Thus we conclude by 1.2. Taking duals, from Lemma 1.2 we obtain the following result.

Lemma 1.4. Let $X$ be a smooth curve of genus $\geq 1$. Fix $A \in \operatorname{Pic}(X)$ and $M_{i} \in \operatorname{Pic}(X), 1 \leq i \leq r-1$, with $\operatorname{deg}(M i)=\operatorname{deg}(A)+1$ for every $i$. Assume $M_{i} \cong M_{k}$ if and only if $i=k$ and $h^{0}(X, \operatorname{Hom}(A, M i))=0$ for every integer $i$ with $1 \leq i \leq r-1$ i.e., assume that there is no $P \in X$ with $M_{i} \cong A(P)$ for some $i$. Set $M:=M 1 \oplus \cdots \oplus M_{r-1}$. Let $E$ be a rank $r$ vector bundle on $X$ fitting in an exact sequence (1). Then $E$ is stable if and only if it has no $M_{i}$ as a direct factor.

By quoting Lemma 1.4 instead of Lemma 1.2 in the proof of Corollary 1.3 we obtain the following result.

Corollary 1.5. Let $X$ be a smooth curve of genus $\geq 1$. Fix $A \in \operatorname{Pic}(X)$ and $M_{i} \in \operatorname{Pic}(X), 1 \leq i \leq r-1$, with $\operatorname{deg}\left(M_{i}\right)=\operatorname{deg}(A)+1$ for every $i$. Assume $M_{i} \cong M_{k}$ if and only if $i=k$ and $h^{0}\left(X, \operatorname{Hom}\left(A, M_{i}\right)\right)=0$ for every integer $i$ with $1 \leq i \leq r-1$ i.e., assume that there is no $P \in X$ with $M_{i} \cong A(P)$ for some $i$. Set $M:=M_{1} \oplus \cdots \oplus M_{r-1}$. Assume $h^{0}(X, A) \geq$ 2 and that each $M_{i}$ is spanned. Fix a linear space $W \subseteq H^{0}(X, M)$ with $\operatorname{dim}(W)=r$ and $W$ spanning $M$. Let $E$ be the general spanned bundle obtained by Construction 1.1 with respect to the vector space $W$. Then $E$ is stable.

Lemma 1.6. Fix a non-split exact sequence

$$
0 \rightarrow H \rightarrow E \rightarrow G \rightarrow 0
$$

of vector bundles on $X$ with $H$ semistable, $G$ stable, $\mu(H)<\mu(G)$ (i.e., $\mu(E)<\mu(G)$ ) and such that $\mu(G)$ is the minimal rational number of the form $a / b$ with $a, b$ integers, $1 \leq b<\operatorname{rank}(E)$, and $a / b>\mu(E)$. Then $E$ is stable.

Proof. Assume $E$ unstable and take a proper subbundle $T$ of $E$ with slope $\mu(T) \geq \mu(E)$. Taking such $T$ with minimal rank we may assume that $T$ is stable. First assume $\mu(T)>\mu(E)$. Since $1 \leq \operatorname{rank}(T)<\operatorname{rank}(E)$, we have $\mu(T) \geq \mu(G)$ by the assumption on $\mu(G)$. Since $G$ is stable, either the induced map $\alpha: T \rightarrow G$ is zero or it is surjective. In the former case we have $T \subseteq H$. Since $H$ is semistable, we obtain $\mu(T) \leq \mu(H)<\mu(G)$, contradiction. Now assume that the induced map $\alpha: T \rightarrow G$ is surjective. If $\alpha$ is an isomorphism, then (4) splits, contradiction. If $\alpha$ is surjective but not 
an isomorphism, we have $\mu(T)<\mu(G)$ because $T$ is stable. Hence we obtain a contradiction and prove that $\mu(T)=\mu(E)$, i.e., up to now we checked the semistability of $E$. If $\mu(T)=\mu(G)$ the map $\alpha$ is either 0 or surjective because $G$ has no proper subsheaf of slope $\beta$ with $\mu(E) \leq \beta<\mu(G)$ by the property of $\mu(G)$ and the stability of $G$. As above we obtain a contradiction and prove the lemma. A similar, but easier, proof give the following result.

Lemma 1.7. Fix a non-split exact sequence (4) of vector bundles on $X$ with $H$ and $G$ semistable, $\mu(H) \leq(G)$ (i.e., $\mu(E) \leq \mu(G)$ ) and such that $\mu(G)$ is the minimal rational number of the form $a / b$ with $a, b$ integers, $1 \leq b<\operatorname{rank}(E)$, and $a / b \geq \mu(E)$. Then $E$ is semistable.

Remark 1.8. By Riemann-Roch we have $h^{1}(X, \operatorname{Hom}(G, H))=\operatorname{rank}(G)$. $\operatorname{rank}(H)(g-1+\mu(G)-\mu(H))+h^{0}(X, \operatorname{Hom}(G, H)) \geq \operatorname{rank}(G) \cdot \operatorname{rank}(H)(g-$ $1+\mu(G)-\mu(H))$ and hence under the assumptions of Lemmas 1.6 and 1.7 for any fixed $G$ and $H$ we always have non-split exact sequences (4).

Lemma 1.9. Let $H$ and $G$ spanned vector bundles on $X$ with $H$ semistable, $G$ stable, $\mu(H)<\mu(G)$ (i.e., $\mu(E)<\mu(G)$ ) and such that $\mu(G)$ is the minimal rational number of the form $a / b$ with $a, b$ integers, $1 \leq b<\operatorname{rank}(E)$, and $a / b>\mu(G)$. Assume $h^{0}(X, \operatorname{Hom}(H, G))=0$. Then there exists a spanned stable vector bundle $E$ on $X$ fitting in the exact sequence (4) and with $h^{0}(X, E)=h^{0}(X, H)+h^{0}(X, G)$.

Proof. Apply Construction 1.1 taking $M:=G, V:=H^{0}(X, G)$ and $A:=$ $H$. Let $U:=\operatorname{ker}\{G, V\}$ be the associated kernel bundle. For every $j \in$ $H^{0}(X, \operatorname{Hom}(U, H))$ the bundle $\operatorname{coker}(u(j))$ fits as middle term in an exact sequence (4). By Lemma 1.6 it is sufficient to show that for general $j \in$ $H^{0}(X, \operatorname{Hom}(U, H))$, the corresponding exact sequence does not split. Fix $j \in H^{0}(X, \operatorname{Hom}(U, H))$ such that $\operatorname{coker}(u(j)) \cong H \oplus G$. Hence $j$ induces an exact sequence

$$
0 \rightarrow U \rightarrow H \oplus W \rightarrow H \oplus G \rightarrow 0 .
$$

Since $h^{0}(X, \operatorname{Hom}(H, G))=0$ and for every homomorphism $f: W \oplus H \rightarrow$ $H \oplus G, f \mid\{0\} \times H$ factors through the inclusion of $H \oplus\{0\}$ into $H \oplus G$.

Hence we obtain $j=0$. Thus to show that for general $j \operatorname{coker}(u(j)) \neq$ $H \oplus G$ it is sufficient to show that $h^{0}(X, \operatorname{Hom}(U, H))>0$. This is true because both $U^{*}$ and $H$ are spanned.

Remark 1.10. The proof of 1.9 works in the set-up of Lemma 1.7 and gives the existence semistable non-split spanned bundles fitting in (4) even if $G$ is only assumed to be semistable and $\mu(H)=\mu(G)$.

Proof of Theorem 0.1. By Corollaries 1.3 and 1.5 for every integer $r \geq 2$ Theorem 0.1 is true for the integers $d=r k+1$ and $d=r(k+1)-1$. In particular 0.1 is true for $r=2$. Fix $r \geq 3$. Assume $r k+2 \leq d \leq r(k+1)-2$ 
and that Theorem 0.1 is true on $X$ for all ranks $r^{\prime}<r$. We take integers $r^{\prime}, r^{\prime \prime}, a, b$ with $r^{\prime}>0, r^{\prime \prime}>0, r^{\prime}+r^{\prime \prime}=r, a+b=d$ and such that the pair of rational numbers $\left(a / r^{\prime}, b / r^{\prime \prime}\right)$ satisfies the assumption of the pair $(\mu(H), \mu(G))$ of Lemma 1.6. We apply the inductive assumption on $r$ to find stable spanned bundles $H, G$ with $\operatorname{deg}(H)=a, \operatorname{rank}(H)=r^{\prime}, \operatorname{deg}(G)=b$ and $\operatorname{rank}(G)=r^{\prime \prime}$. Then we apply Corollary 1.7. It is easier to prove the existence of spanned semistable bundles. Indeed we have the following result.

Proposition 1.11. Let $X$ be a smooth projective curve of genus $g \geq 2$. Fix integers $r, k, m$ with $r \geq 2, k \geq 2$ and $m>0$. Assume the existence of line bundles $L_{i} \in \operatorname{Pic}^{k}(X), 1 \leq i \leq r, M_{u} \in \operatorname{Pic}^{k+1}(X), 1 \leq u<r$, with the following properties:

(a) Every $L_{i}, 1 \leq i \leq r$, and every $M_{u}, 1 \leq u<r$, is spanned;

(b) $h^{0}\left(X, L_{i}\right) \geq m$ for every integer $i$ with $1 \leq i \leq r$ and $h^{0}\left(X, M_{u}\right) \geq$ $m+1$ for every integer $u$ with $1 \leq u<r$;

(c) no two of the line bundles $L_{i}, 1 \leq i \leq r$, are isomorphic;

(d) no two of the line bundles $M_{u}, 1 \leq u<r$, are isomorphic;

(e) we have $h^{0}\left(X, \operatorname{Hom}\left(L_{i}, M_{u}\right)\right)=0$ for every pair $(i, u)$ with $1 \leq i \leq r$ and $1 \leq u<r$, i.e., for every pair $(i, u)$ there is no $P \in X$ with $M_{u} \cong L_{i}(P)$.

Then for every integer $d$ with $r k \leq d<r(k+1)$ there exists a rank $r$ spanned semistable vector bundle $E$ on $X$ with $\operatorname{deg}(E)=d$ and $h^{0}(X, E) \geq$ $m r$.

Proof. If $d=r k$, set $E=\oplus_{1 \leq i \leq r} L_{i}$. If $r k<d<r(k+1)$, just apply Theorem 0.1. However, notice that the proof of semistability in 1.6 (and hence for Theorem 0.1) was much easier than the proof of stability.

\section{References}

[1] E. Arbarello, M. Cornalba, Ph. Griffiths and J. Harris, Geometry of Algebraic Curves, I, Springer, Berlin-Heidelberg-New York-Tokyo, 1985, MR 86h:14019, Zbl 0559.14017.

[2] E. Ballico, On the Brill-Noether theory of spanned vector bundles on smooth curves, Serdica Math. J., 25 (1999), 115-130, MR 2001g:14056, Zbl 0946.14019.

[3] M. Coppens, The existence of base point free linear systems on smooth plane curves, J. Algebraic Geometry, 4 (1995), 1-15, MR 95m:14020, Zbl 0842.14020.

[4] M. Coppens and T. Kato, The gonality of smooth curves with plane models, Manuscripta Math., 70 (1990), 5-25, MR 92a:14027a, Zbl 0725.14005, and Correction, Manuscripta Math., 71 (1991), 337-338, MR 92a:14027b.

[5] M. Coppens, C. Keem and G. Martens, The primitive length of a general k-gonal curve, Indag. Math., (N.S.) 5 (1994), 145-159, MR 95c:14021, Zbl 0845.14019.

[6] V. Mercat, Le problème de Brill-Noether et le thèoréme de Teixidor, Manuscripta Math., 98 (1999), 75-85, MR 2000d:14035, Zbl 0976.14021. 
Received September 29, 1999 and revised January 18, 2002. The author was partially supported by MURST and GNSAGA of INdAM (Italy) .

Department of Mechanics and Mathematics

UNIVERSITY OF TRENTO

38050 Povo (TN), ITLAy

E-mail address: ballico@science.unitn.it 